\title{
An Ensemble of Learning Machines for Quantitative Analysis of Bronze Alloys
}

\author{
Eleonora D'Andrea and Beatrice Lazzerini \\ Department of Information Engineering \\ University of Pisa \\ Largo Lucio Lazzarino, 56122 Pisa, Italy \\ eleonora.dandrea@for.unipi.it, b.lazzerini@iet.unipi.it
}

\begin{abstract}
We deal with the determination of the composition of bronze alloys measured through Laser-Induced Breakdown Spectroscopy (LIBS) analysis. The relation between LIBS spectra and bronze alloy composition, represented by means of the concentrations of constituting elements, is modeled by adopting an ensemble of learning machines, fed with different inputs. Then, the combiner computes the final response. The results obtained on the test set show that the ensemble model manages to determine the composition of alloy samples with mean squared error of about $6.5310^{-2}$.
\end{abstract}

Keywords-ensemble; feature selection; Laser-Induced Breakdown Spectroscopy; neural networks

\section{INTRODUCTION}

Recently, the advances in analytical science and instrumentation technology have led to the improvement in analysis techniques to determine the elemental composition of a sample, e.g., in biomedical, environmental, archaeological, and cultural heritage fields. Elemental analysis may be qualitative, if it is devoted to determine what elements are present in the sample, or quantitative, if it deals with detecting the quantity of each element composing the sample. In the analysis of samples it is of the utmost importance to assure their preservation and integrity. In addition, samples are not always transportable to the laboratory for the analysis. For all these reasons it is necessary to develop efficient, nondestructive, and applicable in situ techniques [1]

Laser-Induced Breakdown Spectroscopy (LIBS) is a spectroscopic technique frequently applied to identify the absolute concentrations of chemical elements, thus performing both qualitative and quantitative elemental analysis of several types of samples, such as soils, metal alloys, and rocks [2]. LIBS represents a valid fast and non-destructive alternative to other spectroscopic, mass spectrometric, or X-ray techniques. Further, it can be used with samples of any shape in liquid, gas or solid state, can be applied in situ, and it does not need a preliminary sample treatment. During LIBS analysis (Fig. 1), a high power laser beam is focused for a few seconds on the sample surface so as to cause the ablation of an amount of mass of the order of nanograms or picograms, thanks to fast increase of temperature in the heated region. The ablated mass interacts

This work was supported by the project "Analisi di dati sensoriali: dai sensori tradizionali ai sensori sociali", funded by "Progetti di Ricerca di Ateneo PRA 2016" of the University of Pisa. with the laser pulse and produces a high-temperature plasma on the sample surface. Afterwards, the plasma expands and cools so that the characteristic atomic emission lines of the elements can be observed. More precisely, the light emitted by the plasma is composed of discrete spectral peaks collected with a spectrograph, and is processed to determine the composition of the sample, given that each chemical element is identified by univocal LIBS spectral peaks. The principal drawbacks of LIBS analysis are limited precision and sensitivity, which negatively influence accuracy [3].

In the following, a few classical approaches for LIBS quantitative analysis are briefly recalled. The typical approach uses calibration curves (CC-LIBS) [4]. The calibration curves (intensity of the emission line vs. concentration of the related element) are obtained according to appropriate reference standards, and then employed to detect the composition of similar samples. The principal disadvantage of this method is the requirement for reference calibration samples. Another method to solve this problem, is the Calibration-Free LIBS (CF-LIBS) [5]. It takes into account the plasma temperature, and the electron number density, and assumes stringent experimental conditions. Its disadvantages are the requirements of a long temporal analysis and of the detection of one line for each element in the plasma with known atomic data. Cavalcanti et al. [6] proposed the One-Point Calibration method (OPCLIBS), which gives the best results and requires the use of one reference sample to calibrate the model.

Another issue concerning LIBS analysis is the highdimensionality of the input space. LIBS spectra are generally represented by means of $N$ spectral lines (where $N$ is of the order of thousands). In fact, the use of too many features tends to increase complexity and decrease the accuracy of the analysis, thus making dimension reduction a necessary preliminary phase. Typically, Partial Least Squares (PLS) Regression [7], or Principal Component Analysis are adopted so as to extract important information from the spectrum, with the aim of achieving the required accuracy and maintaining the lowest possible complexity of the approximation. For this reason, feature selection (FS) is typically adopted to decrease the number of considered input intensities of spectral lines to the minimum value $n$ (with $n<N$ ). Frequently used methods for FS are forward feature selection (FFS) [8] and genetic algorithms (GA) [9]. E.g., the authors in [10] deal with 
classification of explosives with diverse composition using LIBS data. They reduce the large dimensionality of LIBS spectra by selecting the smallest possible subset of discriminatory features in two different ways: i) choice of the spectral areas based on a priori knowledge of the chemical composition of the samples; ii) spectral areas determined by a GA. In both cases, they achieve classification performance higher than that obtained by corresponding algorithms that employ the full spectral data. However, the number of selected features, though being an order of magnitude smaller than the original features, is still sensibly high.

Recently, computational intelligence techniques, and in particular artificial neural networks (NNs) and fuzzy inference systems (FISs), have been successfully employed in [11]-[15] for quantitative analysis of LIBS spectra. In particular, ensembles of intelligent machines are typically adopted in the literature to solve problems concerning classification and regression through the appropriate combination of the outputs of independent learning systems. These systems independently perform the same task and their responses are combined in order to obtain a global response that is better than each individual response [8], [16]-[19]. The performance of such an ensemble is affected by, e.g.,: i) the adoption of different training sets, input representation, and training algorithms, ii) the system architecture, iii) the combination criterion. Actually, the appropriate combination of a collection of imperfect experts enables compensating the limitations of the single experts [16], thus obtaining higher accuracy with respect to the simple choice of the best expert. Even if the most used combining strategy is the averaging method [20], the results can be combined in other ways, such as minimum, maximum, median, weighted average, e.g., taking into account the experts' performances.

In this paper we aim to detect the elemental composition of bronze alloy samples, based only on a subset of the spectral lines describing the samples. For this reason, we propose an ensemble of independent learning machines, with inputs chosen based on different FS procedures. The paper is organized as follows. Sections II and III describe, respectively, the theoretical background on NNs, FISs, FS and the LIBS measurements of bronze alloy data used in the experiments. Section IV introduces the proposed ensemble model to detect the composition of alloy samples. Section V and VI present, respectively, the experimental results, and the conclusions.

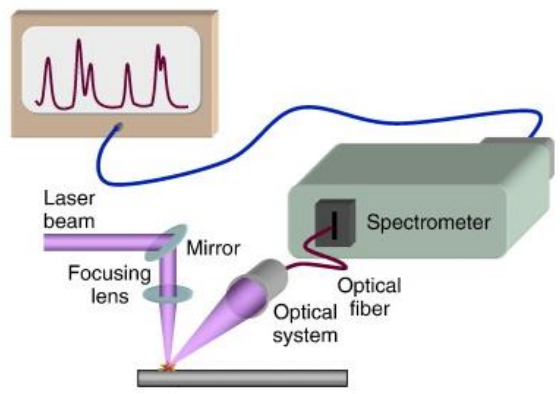

Fig. 1. LIBS analysis [12].

\section{THEORETICAL BACKGROUND}

In the following we briefly describe the basic theory about the machine learning techniques involved in this work.

NNs are intelligent systems which show the remarkable capability to learn from examples. They are able to approximate each input-output relationship with any degree of accuracy. A frequently adopted feed-forward NN model is the radial basis function (RBF) NN. An RBF NN [21] has, in its most typical form, an input layer, which receives input signals, a linear/non-linear output layer, which produces the outputs, and one non-linear hidden layer. The activation function of each hidden neuron is a radial basis function (most often the Gaussian function), whose response decreases, or increases, monotonically with distance from the function center. The training of RBF networks consists in determining the centers and spreads of the radial basis functions, and the weights that connect hidden neurons to output neurons.

Even though NNs are frequently adopted as individual systems to approximate input-output relationships, they have also been employed successfully in combination with other techniques, in particular fuzzy systems, to solve fitting or classification problems. The resulting neuro-fuzzy hybrid system allows exploiting at the same time the capability of NNs of learning from examples and the interpretability of the fuzzy rules. A well-known neuro-fuzzy hybrid system is the Adaptive Neuro-Fuzzy Inference System (ANFIS) [22], a Sugeno fuzzy inference system which employs a hybrid learning process to automatically determine, from an inputoutput data set, the fuzzy system's membership function parameters, and the fuzzy rules. The typical learning process of ANFIS combines least-squares and backpropagation gradient descent methods.

Regarding feature selection, FFS is a well-known method for input dimensionality reduction. It starts by considering and evaluating, based on a given criterion function, all sets containing a single feature to approximate the input-output relationship. Then, FFS generates the best subset containing two features (the one selected in the previous step and another feature from the remaining ones), three features, and so on. The final subset is the best among all the subsets generated. Another well-known method used for feature selection is based on GAs [9]. A GA is an optimization algorithm based on the mechanics of natural selection and genetics, which describes the candidate solution to the optimization problem as an individual (or chromosome) having a set of characteristics (i.e., free parameters of the problem) and then evolves a population of individuals toward the optimal solution for the problem, according to a fitness function, which measures the quality of each individual in approximating the functional relationship between inputs and outputs. Generally, GAs start with a randomly generated initial population of chromosomes, representing candidate solutions to the problem, and evolve toward populations with chromosomes having, on average, a better fitness. This is achieved thanks to the application of genetic operators like crossover, mutation, and selection. On the GA convergence, the chromosome associated with the best fitness value is typically chosen as the optimal solution. 


\section{BRONZE ALLOYS DATASET}

The dataset used in this work was collected by analyzing 6 different physical samples of modern bronze alloys with the concentrations of the four constituting chemical elements shown in Table I: the majority element Copper $(\mathrm{Cu})$, Zinc $(\mathrm{Zn})$, Tin $(\mathrm{Sn})$, and Lead $(\mathrm{Pb})$. In the following, the word "sample" refers to a particular modern bronze alloy, that is, a particular composition of 4 chemical elements. Further, the word "spectrum" is used to indicate a particular instance of a sample. Stated in other words, a sample represents a set of spectra. 150 spectra for each sample were collected and elaborated, thus obtaining a total of 900 spectra. The LIBS spectra represent the intensity of the LIBS signal at 3606 wavelength or spectral lines (in the range $200 \div 900 \mathrm{~nm}$ ), so a spectrum is represented in $\mathfrak{R}^{3606}$. With the aim of having all the emission lines on the same intensity scale, we preprocessed the data, by normalizing the intensity values in $[0 \div 1]$ based on the max-min formula. Fig. 2 shows a typical spectrum. As stated before, each spectral line indicates weather the corresponding chemical element is present (or absent) in the sample, and the intensity of the spectral line suggests the corresponding concentration of the chemical element.

The data introduced above are the same as those used in [19] but with a different purpose, namely, the prediction of the chemical composition of unknown modern bronze alloy samples.

\section{TABLE I. COMPOSITON OF THE BRONZE ALLOYS SAMPLES}

\begin{tabular}{|c|c|c|c|c|}
\hline \multirow{2}{*}{$\begin{array}{c}\text { Sample } \\
\text { Id }\end{array}$} & \multicolumn{4}{|c|}{ Composition (\% in weight) } \\
\cline { 2 - 5 } & Copper & Zinc & Tin & Lead \\
\hline S160 & 83.6 & 1.9 & 11.4 & 3.1 \\
\hline $\boldsymbol{S 1 6 1}$ & 87.1 & 5 & 5.5 & 2.4 \\
\hline $\boldsymbol{S 1 6 2}$ & 90.9 & 0.3 & 7.8 & 1 \\
\hline $\boldsymbol{S 1 6 3}$ & 84.1 & 1.4 & 10.5 & 4 \\
\hline $\boldsymbol{S 1 6 4}$ & 85.7 & 9.5 & 3.6 & 1.2 \\
\hline $\boldsymbol{S 1 6 5}$ & 87.3 & 3.7 & 6.6 & 2.4 \\
\hline
\end{tabular}

\section{Proposed ARCHITECTURE}

We chose to develop the system as an ensemble of learning machines, by performing the following two main steps:

1) development of $K(K \geq 2) \mathrm{NN}$-based experts performing the same task. The task consists in approximating the functional relationship between each spectrum represented in $\mathfrak{R}^{3606}$ (the 3606 spectral lines) and the corresponding composition represented in $\mathfrak{R}^{4}$ (the concentrations of the four chemical elements in the alloy). Each expert is a model appropriately trained to perform the input-output mapping (see Sections IV.A, IV.B, and IV.C).

More in detail, this step requires a preliminary objective, i.e., to solve a high-dimensional feature selection problem, with the aim of choosing the most significant spectral lines of the LIBS spectrum so as to significantly decrease the problem dimensionality, while preserving a highly accurate approximating model. Thus, step 1) consists in performing two sub-steps:

a) an FS process tailored to each expert (the FS parameters for each expert are summarized in Table II), which selects the appropriate set of features $S F_{i}$, $i=1, \ldots, K$, used to represent the inputs to the expert (thus, the inputs to the experts may be different from each other), and

b) the setup of the model parameters (e.g., number of hidden neurons) based on the features selected;

2) choice of the optimal way of combining the outputs coming from the experts into a single response (see Section IV.D).

In the experiments we developed an ensemble of $K=3$ experts, namely, two RBF NNs, and one ANFIS system. Referring to the last, actually we built four ANFIS models, one for each concentration value to be estimated, as the classical implementation of ANFIS is single-output.

The ensemble is first trained and tuned on a subset of the spectra (training set), then it is tested on the remaining spectra (test set) in order to check the behavior of the ensemble on previously unseen spectra. We build the training set and test set as follows. The training set corresponds to about $80 \%$ of the available spectra (spectra randomly selected within each sample in order to have the same number of spectra for each sample), while the test set corresponds to the remaining spectra. The following sub-sections describe the development of the learning machines, and of the combiner. Fig. 3 depicts the architecture of the proposed ensemble model developed within the Matlab ${ }^{\circledR}$ environment. The details are given in the following.

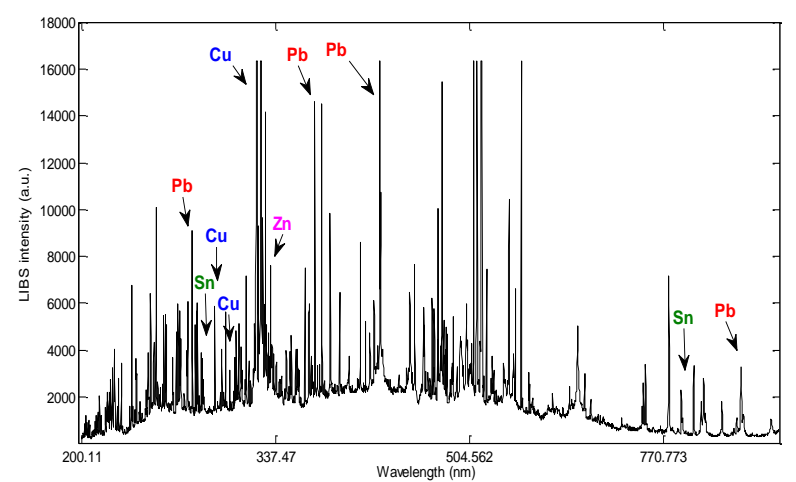

Fig. 2. A LIBS spectrum of sample S160 showing some associations between spectral line and chemical element.

\section{A. First Expert}

The first expert ( $r b f_{-} n n_{1}$ in Fig. 3) is an RBF network whose inputs are the features selected with an FFS process applied to the training set expressed in terms of the original 3606 features.

At each step, FFS selects the optimal subset of features able to minimize the error of the criterion function, which is an RBF network whose inputs are, at each step, the selected features, and whose outputs are the 4 concentrations. We adopted an RBF NN with the Matlab ${ }^{\circledR}$, s default values for the network parameters. The resulting set of selected features is the set of 4 LIBS intensities $S F_{1}=\{914,977,1884,1916\}$ corresponding to the spectral lines (in nm) $\{326.15,334.45,472.05,481.05\}$. We took into account only 4 features, as we observed that after adding the 5-th feature, the Mean Squared Error (MSE) does 
not decrease in a meaningful way

The RBF architecture employed to implement the first expert has one hidden layer, with a maximum number of neurons set to 20,4 inputs (the features previously selected) and 4 outputs. The transfer functions for hidden and output neurons are Gaussian and linear, respectively. An experimental analysis was performed to find out the best value for the spread. We considered values in the range from 0.1 to 1 with step 0.1 , and for each of them, the RBF NN was trained 10 times, by employing the training set. The final best RBF network resulted to be the one with the minimum average MSE on the 10 training sets. We finally found 0.1 as the optimal spread value, and 18 as the optimal number of hidden neurons.

TABLE II. PARAMETERS OF THE FEATURE SELECTION PROCESSES

\begin{tabular}{|c|c|c|}
\hline \multirow{2}{*}{ Expert } & \multicolumn{2}{|r|}{ Feature selection process } \\
\hline & FS kind & Parameters \\
\hline $\begin{array}{c}\text { First } \\
\text { Expert }\end{array}$ & FFS & $\begin{array}{l}\text { Criterion function: RBF NN function (20 } \\
\text { hidden neurons, spread value 1) }\end{array}$ \\
\hline $\begin{array}{l}\text { Second } \\
\text { Expert }\end{array}$ & $\begin{array}{l}\text { GA- } \\
\text { based }\end{array}$ & $\begin{array}{l}\text { Selection: stochastic uniform } \\
\text { Crossover: single-point, probability } 0.1 \\
\text { Mutation: Gaussian } \\
\text { Population size: } 30 \text { chromosomes } \\
\text { Maximum number of generations: } 200 \\
\text { Fitness function: RBF NN function ( } 20 \text { hidden } \\
\text { neurons, spread value } 1)\end{array}$ \\
\hline $\begin{array}{c}\text { Third } \\
\text { Expert }\end{array}$ & FFS & $\begin{array}{l}\text { Criterion function: ANFIS model (grid } \\
\text { partitioning, } 3 \text { triangular membership functions } \\
\text { per variable) }\end{array}$ \\
\hline
\end{tabular}

\section{B. Second Expert}

The second expert is an RBF network ( $r b f_{-} n n_{2}$ in Fig. 3) whose inputs are the spectral lines selected through a GAbased FS process. Due to computational cost reasons, FS was applied on a reduced set of 480 features selected among the 3606 by a human expert and already employed in [6]. Thus, each spectrum is represented in $\mathfrak{R}^{480}$. In addition, we made a further simplification: we chose 10 as the maximum number of features, by adapting the GA mutation function. The representation of the chromosome is a binary-coded vector of 480 genes, where each gene indicates weather the corresponding feature is present or not in the final set. Details about selection, crossover, and mutation operators are summarized in Table II. We set the maximum number of generations to 200, and the population size to 30 chromosomes. The fitness function is an RBF NN-based criterion function. In this case, too, we adopted an RBF NN with Matlab ${ }^{\circledR}$ 's default values for the network parameters. The GA-based FS found the set of 6 LIBS intensities $S F_{2}=\{715,912,977,1155,2560$, $2621\}$ corresponding to the spectral lines (in $\mathrm{nm}$ ) $\{299.56$, $325.88,334.45,357.60,657.01,673.12\}$.

We wish to point out that, though the GA-based FS process was applied, for computational reasons, on a reduced set of features, where the significant information usually lies according to the experts in the field, starting from this reduced set of features does not limit the capability of the system. Indeed the final ensemble, as a whole, actually takes into account all the available features, thus allowing search for relevant information in features not taken into account in this reduced set.

Finally, to choose the final best RBF NN to implement the second expert, we repeated the steps made in the case of the first expert. The final RBF architecture has 6 input variables and 4 output variables. As before, the transfer functions for hidden and output neurons are Gaussian and linear, respectively. The optimal values for the spread and the number of hidden neurons resulted to be 0.1 , and 16 , respectively.

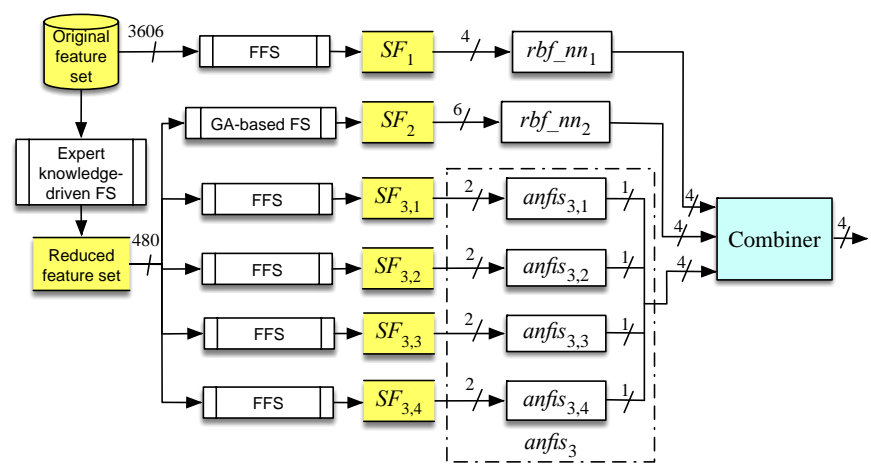

Fig. 3. Architecture of the proposed ensemble of learning machines.

\section{Third Expert}

The third expert is an ANFIS-based model (anfis $s_{3}$ in Fig. 3). More in detail, we developed four ANFIS models, one for each output concentration. We performed four different FS processes on the reduced set of 480 features, by employing FFS. The criterion function of each FFS is the standard ANFIS model (developed using the Matlab ${ }^{\circledR}$ s $s$ default values, i.e., grid partitioning with 3 triangular membership functions per variable), having as inputs, at each step, the selected features, and as output the considered concentration. The four FS processes found the following sets of LIBS intensities: 1) $S F_{3,1}=\{977,1205\}$, corresponding to the set of spectral lines (in $\mathrm{nm}$ ) $\{334.45,364.02\}$ for the Copper concentration; 2) $S F_{3,2}=\{922,976\}$, corresponding to the set of spectral lines (in $\mathrm{nm}) \quad\{327.21,343.32\}$ for the Zinc concentration; 3) $S F_{3,3}=\{976,1727\}$, corresponding to the set of spectral lines (in $\mathrm{nm}$ ) $\{343.32,428.63\}$ for the Tin concentration; and 4) $S F_{3,4}=\{703,977\}$, corresponding to the set of spectral lines (in nm) $\{297.94,334.45\}$ for the Lead concentration. We chose only the first two features selected by FFS because we verified that using a higher number of features did not improve significantly the results, and also to maintain the ANFIS models as simple as possible.

To find the optimal architecture of each of the 4 ANFIS models (anfis $s_{3,1}$, anfis $s_{3,2}$, anfis $s_{3,3}$, anfis $s_{3,4}$ in Fig. 3) which constitute the third expert, we tried the following settings for the generations of the models:

i) grid partitioning with 3 , 4, or 5 membership functions per input, and linear membership functions for the output; we took into account also several membership functions types, i.e., triangular, Gaussian, trapezoidal, and generalized bell-shaped;

ii) subtractive clustering with the default values proposed in Matlab ${ }^{\circledR}$;

iii) fuzzy c-means clustering with 2, 3, 4, or 5 clusters, and Matlab ${ }^{\circledR}, s$ default values for the other parameters. 
Each model architecture was trained 10 times, by employing the training set. The best model architecture for all the ANFIS models resulted to be the one built with the fuzzy $c$ means clustering with 3 clusters.

\section{The Ensemble}

Once the optimal architectures of the three learning machines have been found, the ensemble model can be developed. The development of the ensemble consists in appropriately combining the responses from the 3 experts, related to the 4 concentration values of the elemental composition. Hence the combiner is a function having 12 inputs and 4 outputs, i.e., the ensemble values of the 4 concentrations. Each ensemble concentration value (e.g., Copper) produced by the combiner is the combination of the values of the corresponding concentrations (Copper) produced by the experts. The combination consists in the application of a combining function. We took into account several combining functions, i.e., mean, maximum, minimum, median, weighted average, geometric mean, weighted geometric mean, harmonic mean, Heronian mean.

After the setup with the optimal values of the parameters, each learning machine is trained, and the output from each learning machine is fed as input to the combiner. We tried all the combining functions mentioned above. To keep computational time at an acceptable level, each experiment was repeated 10 times for each possible combining function. The best combining function resulted to be the weighted average, with the sum of weights being unity. The weights of the weighted average were chosen heuristically according to the performance of the corresponding experts during the training process.

\section{EXPERIMENTAL RESULTS}

In the experiments, the error after the combination is computed by comparing the estimated concentrations with the target concentrations of the four chemical elements on the training and test sets. The error produced by the model is computed as the average MSE over $Q$ executions. We chose $Q=30$, as suggested in [17]. Table III shows the obtained results related to the training and test sets in terms of MSE achieved by sample over the 30 trials. Table IV shows the MSE on the training and the test sets over the 30 executions obtained by the experts, and by the ensemble.

As a final remark, we wish to point out that the proposed approach to LIBS quantitative analysis of samples is fast and easy and it overcomes the main drawbacks of the classical approaches, namely, complex calculations of spectral parameters, long times for analysis, need for strict experimental condition or for standard samples for calibration. In addition, it allows performing a fast and automatic analysis of a large number of spectra.

\section{CONCLUSIONS}

This paper has proposed an ensemble of learning machines for determining the elemental composition of bronze alloys represented through LIBS spectra. We performed different FSs depending on the expert, to reduce the input dimensionality. With respect to classical approaches for LIBS analysis, we employ a remarkably limited number of spectral lines to detect the elemental composition. In addition, such a process could be easily automatized, e.g., in industrial applications with strict time requirements in data processing.

\section{ACKNOWLEDGMENT}

We wish to acknowledge Prof. V. Palleschi, Applied Laser Spectroscopy Lab., ICCOM-CNR, Pisa, Italy, for having provided the data used in the experiment.

TABLE III. AVERAGE MSEs by SAMPLE, ACHIEVEd by the ENSEMble.

\begin{tabular}{|c|c|c|c|c|c|c|}
\hline \multirow{2}{*}{} & \multicolumn{5}{|c|}{ Sample Id } \\
\cline { 2 - 7 } & $\mathbf{S 1 6 0}$ & $\mathbf{S 1 6 1}$ & $\boldsymbol{S 1 6 2}$ & $\boldsymbol{S 1 6 3}$ & $\boldsymbol{S 1 6 4}$ & $\boldsymbol{S 1 6 5}$ \\
\hline Training & $1.4 \cdot 10^{-2}$ & $7.7 \cdot 10^{-2}$ & $3.6 \cdot 10^{-2}$ & $4.3 \cdot 10^{-2}$ & $2.93 \cdot 10^{-2}$ & $1.3 \cdot 10^{-2}$ \\
\hline Test & $3.64 \cdot 10^{-2}$ & $8.8 \cdot 10^{-2}$ & $4.9 \cdot 10^{-2}$ & $6.37 \cdot 10^{-2}$ & $6.82 \cdot 10^{-2}$ & $8.5 \cdot 10^{-2}$ \\
\hline
\end{tabular}

TABLE IV. AVERAGE MSES ObTAINED By the EXPERTS, AND By THE ENSEMBLE.

\begin{tabular}{|c|c|c|c|c|}
\hline & First Expert & Second Expert & Third Expert & Ensemble \\
\hline Training & $3.72 \cdot 10^{-2}$ & $1.131 \cdot 10^{-1}$ & $4.442 \cdot 10^{-1}$ & $3.55 \cdot 10^{-2}$ \\
\hline Test & $6.76 \cdot 10^{-2}$ & 2.055 & $4.484 \cdot 10^{-1}$ & $6.53 \cdot 10^{-2}$ \\
\hline
\end{tabular}




\section{REFERENCES}

[1] M. Osorio, L.V. Ponce Cabrera, M.A. Arronte García, T. Flores Reyes, and I. Ravelo, "Portable LIBS system for determining the composition of multilayer structures on objects of cultural value", J. Phys.: Conf. Ser., vol. 274, no. 1, 2011.

[2] K. Melessanaki, M. Mateo, S.C. Ferrence, P.P. Betancourt, and D. Anglos, "The application of LIBS for the analysis of archaeological ceramic and metal artifacts", Appl. Surf. Science, vol. 197-198, pp. 156163, 2002.

[3] J.D. Winefordner, I.B. Gornushkin, T. Correll, E. Gibb, B.W. Smith, and N. Omenetto, "Comparing several atomic spectrometric methods to the super stars: Special emphasis on laser induced breakdown spectrometry, LIBS, a future super star", J. Anal. At. Spectrom., vol. 19, no. 9, pp. 1061-1083, 2004.

[4] Y. Yoon, T. Kim, M. Yang, K. Lee, and G. Lee, "Quantitative analysis of pottery glaze by laser induced breakdown spectroscopy", Microchem. J., vol. 68, pp. 251-256, 2001

[5] A. Ciucci, V. Palleschi, S. Rastelli, A. Salvetti, and E. Tognoni, "New Procedure for Quantitative Elemental Analysis by Laser-Induced Plasma Spectroscopy", Appl. Spectrosc., vol. 53, no. 8, pp. 960-964, 2013.

[6] G.H. Cavalcanti, D.V. Teixeira, S. Legnaioli, G. Lorenzetti, L. Pardini, and V. Palleschi, "One-point calibration for calibration-free laserinduced breakdown spectroscopy quantitative analysis", Spectrochim. Acta B: Atomic Spectrosc., vol. 87, pp. 51-56, 2013.

[7] J.M. Andrade, G. Cristoforetti, S. Legnaioli, G. Lorenzetti, V. Palleschi, and A.A.Shaltout, "Classical univariate calibration and partial least squares for quantitative analysis of brass samples by laser-induced breakdown spectroscopy", Spectrochim. Acta B: Atomic Spectrosc., vol. 65, no. 8, pp. 658-663, 2010.

[8] S.S. Haykin, Neural networks. A comprehensive foundation, 2nd ed., Prentice-Hall, New Jersey, 1999.

[9] J.H. Holland, Adaptation in Natural and Artificial Systems. University of Michigan Press, 1975.

[10] Kumar Myakalwar, A., Spegazzini N., Zhang C., Kumar Anubham S., Dasari R.R., Barman I., \& Kumar Gundawar M., Less is more: Avoiding the LIBS dimensionality curse through judicious feature selection for explosive detection. Sci. Rep. 5, 13169 (2015). doi: 10.1038/srep13169 (2015).

[11] E. D’Andrea, S. Pagnotta, E. Grifoni, S. Legnaioli, G. Lorenzetti, V. Palleschi, and B. Lazzerini, "A hybrid calibration-free/artificial neural networks approach to the quantitative analysis of LIBS spectra", Applied Physics B, vol. 118, no. 3, pp. 353-360, 2015.

[12] E. D'Andrea, B. Lazzerini, V. Palleschi, and S. Pagnotta, "Determining the composition of bronze alloys by means of high-dimensional feature selection and Artificial Neural Networks", in Proc. 2015 IEEE Int. Instrum. and Meas. Tech. Conf. (I2MTC), Pisa, Italy, May 11-14, 2015, pp. 2049-2054.

[13] J. El Haddad, D. Bruyère, A. Ismaël, G. Gallou, V. Laperche, K. Michel, L. Canioni, and B. Bousquet, "Application of a series of artificial neural networks to on-site quantitative analysis of lead into real soil samples by laser induced breakdown spectroscopy", Spectrochim. Acta B: Atomic Spectrosc., vol. 97, no. 1, pp. 57-64, 2014.

[14] P. Inakollu, T., Philip, A.K. Rai, F.Y. Yueh, and J.P. Singh, "A comparative study of laser induced breakdown spectroscopy analysis for element concentrations in aluminum alloy using artificial neural networks and calibration methods", Spectrochimica Acta Part B: Atomic Spectroscopy, vol. 64, no. 1, pp. 99-104, 2009.

[15] E.C. Ferreira, D.M. Milori, E.J. Ferreira, R.M. Da Silva, and L. MartinNeto, "Artificial neural network for Cu quantitative determination in soil using a portable laser induced breakdown spectroscopy system", Spectrochim. Acta Part B: Atomic Spectrosc.,vol. 63, no. 10, pp. 121620, 2008.

[16] A.J.C. Sharkey, "On combining artificial neural nets", Connection Science, vol. 8, pp. 299-314, 1996.

[17] L.I. Kuncheva, Combining pattern classifiers: Methods and algorithms, Wiley Interscience, New Jersey, 2004.

[18] B. Lazzerini, and S.L. Volpi, "Classifier ensembles to improve the robustness to noise of bearing fault diagnosis", Pattern Analysis and Applications, vol. 16, no. 2, pp. 235-251, 2013.

[19] E. D’Andrea, B. Lazzerini, and V. Palleschi, "Combining Multiple Neural Networks to Predict Bronze Alloy Elemental Composition", in Proc. 25th Italian W. on Neural Networks (WIRN), Vietri sul Mare, Salerno, Italy, May 20-22, 2015.

[20] U. Naftaly, N. Intrator, and D. Horn, "Optimal ensemble averaging of neural networks", Network: Comp. in Neural Systems, vol. 8, pp. 283296, 1997.

[21] D.S. Broomhead, and D. Lowe, "Multivariable functional interpolation and adaptive networks", Complex Systems, vol. 2, pp. 321-355, 1988

[22] J.-S.R. Jang, "ANFIS: adaptive-network-based fuzzy inference system", IEEE Trans. Syst. Man Cybern., vol. 23, no. 3, pp. 665-685, 1993. 